\title{
ş \\ Subradiant spontaneous undulator emission through collective suppression of shot noise
}

\author{
D. Ratner, ${ }^{1, *}$ E. Hemsing, ${ }^{1, \dagger}$ A. Gover, ${ }^{2}$ A. Marinelli, ${ }^{1}$ and A. Nause ${ }^{3}$ \\ ${ }^{1}$ SLAC, Menlo Park, California \\ ${ }^{2}$ Tel-Aviv University, Tel-Aviv, Israel \\ ${ }^{3}$ UCLA, Los Angeles, California \\ (Received 10 December 2014; published 21 May 2015)
}

\begin{abstract}
The phenomenon of Dicke's subradiance, in which the collective properties of a system suppress radiation, has received broad interest in atomic physics. Recent theoretical papers in the field of relativistic electron beams have proposed schemes to achieve subradiance through suppression of shot noise current fluctuations. The resulting "quiet" beam generates less spontaneous radiation than emitted even by a shot noise beam when oscillating in an undulator. Quiet beams could have diverse accelerator applications, including lowering power requirements for seeded free-electron lasers and improving efficiency of hadron cooling. In this paper we present experimental observation of a strong reduction in undulator radiation, demonstrating the feasibility of noise suppression as a practical tool in accelerator physics.
\end{abstract}

DOI: 10.1103/PhysRevSTAB.18.050703

PACS numbers: 29.27.-a, 29.20.Ej, 41.60.Cr

\section{INTRODUCTION}

Dicke's seminal work on superradiance [1] describes the process of controlling radiation through a system's collective properties; in Dicke's superradiance the cooperative emission of e.g., dipoles radiating in phase increases the total radiation, while in subradiance, antiphasing of the dipoles leads to cancellation between the radiation fields and thus reduced radiation. Experimentally, superradiance is easier to observe, but recently focus has turned increasingly to subradiance as well (see e.g., [2-5]). Dicke's results have received most attention from the field of atomic physics [3-5], but subradiance is also directly applicable to relativistic free electrons; e.g., a microbunched electron beam emits superradiantly when oscillating transversely in a magnetic undulator, with the intensity enhanced relative to spontaneous radiation by the number of coherent electrons [6,7]. In the opposite regime, homogenization of the beam on the scale of the radiation wavelength results in destructive interference and consequently subradiance, i.e., lower intensity than expected from spontaneous radiation. In practice, beam homogenization corresponds to suppression of random shot-noise current fluctuations and recent theoretical proposals [8-11] and experimental demonstrations $[12,13]$ have investigated various means of shot-noise suppression.

\footnotetext{
*dratner@slac.stanford.edu

†ehemsing@slac.stanford.edu
}

Published by the American Physical Society under the terms of the Creative Commons Attribution 3.0 License. Further distribution of this work must maintain attribution to the author $(s)$ and the published article's title, journal citation, and DOI.
Undulator subradiance has direct applications in the field of free-electron lasers (FELs). Following recent shortwavelength seeding demonstrations [14] there is increasing interest in pushing seeding to the $\mathrm{x}$-ray regime. Promising seeding schemes including high gain harmonic generation $[15,16]$, echo-enabled harmonic generation $[17,18]$, and direct seeding with high harmonic generation [19] are all limited by the need for a high-power, short-wavelength seed that can outcompete the self-amplified spontaneous emission (SASE) [20] process in the startup of an FEL [21]. However, subradiance has the potential to extend the shortwavelength limit; spontaneous undulator radiation competes with the external seed laser to kick-start the FEL process, so decreasing the spontaneous radiation reduces the required seed power and opens the possibility of seeding at shorter wavelengths. Shot noise suppression also has other potential applications, such as cooling high energy hadrons [22,23], avoiding the microbunching instability (MBI) [24-27], and novel FEL schemes [28].

Previous observations of shot noise suppression in accelerators were only proof-of-principle experiments, using optical transition radiation (OTR) as a diagnostic $[12,13]$, and only demonstrated weak suppression. Moreover, OTR has limited applications and its emission pattern is null on axis. By contrast, undulator emission has direct relevance to short-wavelength FEL seeding and has potential to reach a higher degree of suppression, especially on axis in the beam propagation direction. In this paper we describe the first demonstration of coherent reduction in undulator radiation as well as direct measurement of the angular dependence of the suppression. This measurement represents the first demonstration of Dicke's subradiance of dipole radiation in the context of free electrons. 
TABLE I. Parameter list for experimental conditions.

\begin{tabular}{lc}
\hline \hline Experimental parameters & \\
\hline Beam charge & $25 \mathrm{pC}$ \\
Final beam energy & $120 \mathrm{MeV}$ \\
Gun phase & -5 to $-19.5 \mathrm{deg}$ \\
Peak current & $5-20 \mathrm{~A}$ \\
Normalized emittance $(\mathrm{x}, \mathrm{y})$ & $1 \mu \mathrm{m}$ \\
Slice energy spread & $1.5 \mathrm{keV}$ \\
$\mathrm{C}_{0}$ dispersion $\left(R_{56}\right)$ & $0-8 \mathrm{~mm}$ \\
Interaction beam size $\left(\sigma_{i}\right)$ & $\sim 1 \mathrm{~mm}$ \\
Undulator beam size $\left(\sigma_{u}\right)$ & $\sim 100 \mu \mathrm{m}$ \\
Undulator K parameter $(K)$ & 1.8 \\
Number of undulator periods & 10 \\
Radiation wavelength $\left(\lambda_{r}\right)$ & $800 \mathrm{~nm}$ \\
\hline \hline
\end{tabular}

\section{NOISE SUPPRESSION CONCEPT}

The experimental scheme follows the dispersive method of [10]: space charge (Coulomb) interaction occurs in a region of length $L_{a}$, creating an energy modulation, and a subsequent magnetic chicane of dispersive strength $R_{56}$ generates a corresponding density modulation. Note that this model is identical to that of MBI (see e.g., [29] for an overview). A useful metric for the amplitude of noise suppression at wave number $k$ is the noise factor, $F(k)$, which is a relevant parameter for a number of phenomena, notably the startup of a SASE FEL [20]. We define the noise factor as

$$
F(k) \equiv \frac{1}{N} \sum_{j, l} e^{i k\left(z_{j}-z_{l}\right)}
$$

where the double sum is over all $N$ electrons in the beam and $z_{j}$ is the longitudinal position along the bunch of the $j$ th electron. Using a simple $1 \mathrm{D}$ model we find that for high frequencies $\left(k \sigma_{i} / \gamma \gg 1\right.$, for interaction beam radius $\sigma_{i}$ and relativistic factor $\gamma$ ) and vanishing energy spread, we can write the noise factor as [10]

$$
F(k) \approx 1-2 \Upsilon+\Upsilon^{2}
$$

where

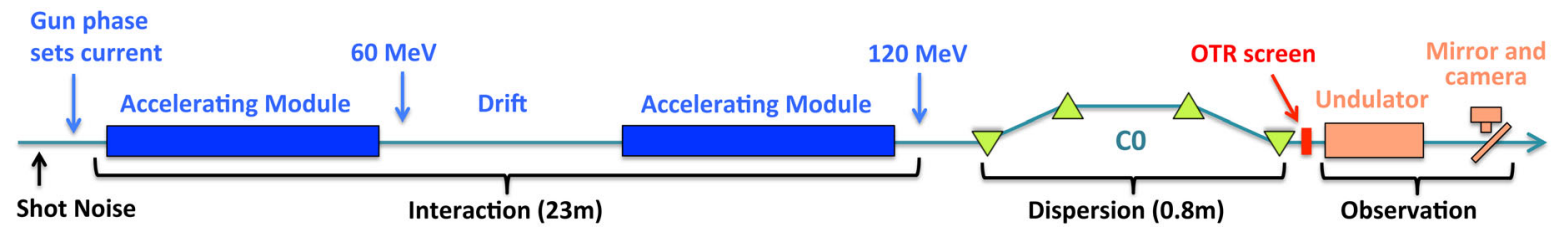

FIG. 1. Experimental schematic: The electron beam's Coulomb forces produce energy modulations in the interaction region. Then passing through the chicane dispersion results in a "quiet" electron beam, which emits subradiantly in an undulator. As a control, an OTR screen can be inserted after the chicane to scramble the electrons and reset a shot noise distribution. with longitudinal particle density $n_{0}$ and classical electron radius $r_{e}$. Matching the dispersive strength, $R_{56}$, to the interaction strength, $n_{0} A$, produces $\Upsilon=1$ and sends the noise factor $F(k) \rightarrow 0$, generating a "quiet" beam. When this quiet beam passes through an undulator, we then expect a corresponding decrease in undulator radiation, i.e., subradiance.

\section{EXPERIMENTAL SETUP}

Experimental work was carried out at the Next Linear Collider Test Accelerator (NLCTA) beam line [18,30] using the parameters of Table I. Figure 1 shows a schematic of the experiment. Space charge interaction between particles occurs during the acceleration and drift sections totaling $23 \mathrm{~m}$ in length. The " $\mathrm{C}_{0}$ " chicane allows control of $R_{56}$ to search for subradiance. At full acceleration, the electrons reach $120 \mathrm{MeV}$, which results in radiation at $\lambda_{r}=800 \mathrm{~nm}$ emitted by a ten-period undulator. A MantaG-125 camera is focused to infinity to capture the far-field radiation profile (power as a function of emitted angle). A low pass filter blocks radiation below $800 \mathrm{~nm}$ so that we only observe emission at the undulator's fundamental wavelength.

Scanning the $R_{56}$ of chicane $\mathrm{C}_{0}$ results in a quadratic dependence in the undulator radiation as a function of $R_{56}$, as expected from Eq. (2). Sample images and results of the scan are given in Figs. 2 and 3. In the scans we find the observed undulator radiation decreases quadratically to the point of maximum suppression. As $R_{56}$ increases further the beam enters the MBI regime (see e.g., [29]) and eventually reaches as much as a factor of 8 above the shot noise level. The $R_{56}$ strength required for suppression is proportional to the strength of the interaction upstream, so changing $n_{0}$ adjusts the point of maximum suppression [Eq. (3)]. Experimentally we use the gun phase to control velocity bunching of the beam, and thus the peak current; zero degrees corresponds to the crest of the rf pulse (approximately 20 A peak current), and moving the phase in the negative direction decompresses the beam and lowers the peak current. Figure 3 shows the point of maximum suppression for three different gun phases. 


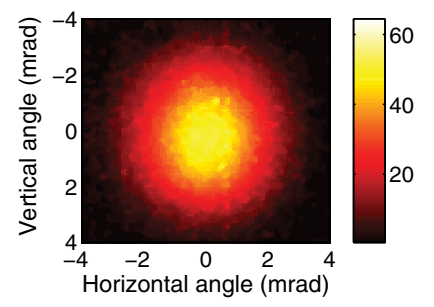

(a)

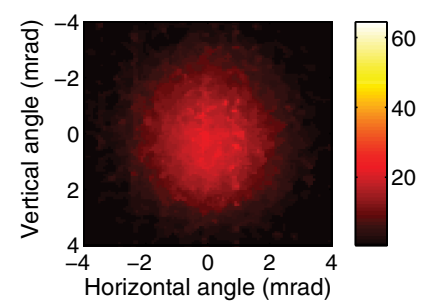

(b)
FIG. 2. Averaged far-field images of spontaneous undulator radiation for (a) $R_{56}=0.1 \mathrm{~mm}$ and (b) $R_{56}=2.2 \mathrm{~mm}$ when suppression is maximal. The color scale shows intensity (arbitrary units). Gun phase is at $-14 \mathrm{deg}$.

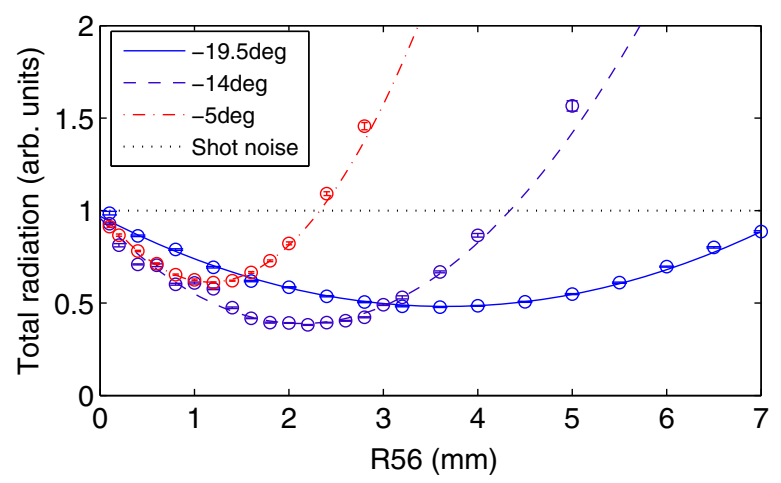

FIG. 3. Scan of undulator radiation amplitude vs $R_{56}$. Colors correspond to different gun phase setting (and thus peak current), and lines are quadratic fits to the data. At larger peak currents, the interaction is stronger, leading to suppression at smaller $R_{56}$ values. Shot noise level (dotted line) is determined by inserting an OTR screen. (The $-5 \mathrm{deg}$ case uses the $-14 \mathrm{deg}$ case normalization.)

\section{DETERMINING THE SHOT NOISE LEVEL}

The dispersive noise suppression model assumes that the particles are frozen longitudinally in the modulation region, and experience no energy modulation in the dispersive region. The chicane is a factor of 30 shorter than the accelerator, so the second assumption is safe. However, the first assumption may not be satisfied strongly, and it is possible that the particles have gone through a portion of a plasma oscillation before reaching the chicane [31,32]. In this case, even with $R_{56}=0 \mathrm{~mm}$, we would expect some degree of subradiance. We can estimate the plasma phase advance from results of Fig. 3; the three gun phase examples correspond to different plasma frequencies, so the fact that the three $R_{56}=0.1 \mathrm{~mm}$ measurements match within roughly $10 \%$ suggests that when the chicane is off, the dashed lines in Figs. 3 and 4 correspond to the uncorrelated, shot noise level.

As a second experimental check of the shot noise level, we insert a screen consisting of $50 \mathrm{~nm}$ of aluminum on a $2 \mu \mathrm{m}$ backing of nitrocellulose (installed as an OTR diagnostics screen) into the path of the beam following

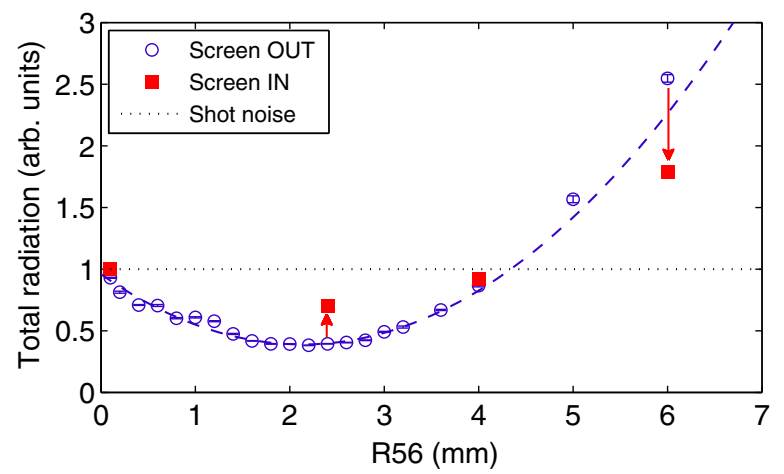

FIG. 4. Intensity on the camera as a function of chicane $R_{56}$ at -14 deg phase with OTR screen OUT (blue circles) and screen IN (red squares). The dotted black line shows the inferred shot noise level. The screen shifts intensity towards the shot noise level, but does not fully suppress coherent effects.

the chicane but upstream of the undulator (Fig. 1). This OTR screen induces an additional $\sim 2 \mathrm{mrad}$ angular spread in the electron beam [see e.g., Eq. (7), Sec. 3.3.1 from [33]]. The larger beam emittance washes out noise suppression and microbunching alike by both scrambling particle positions [34] and expanding the beam size in the undulator (which reduces transverse coherence, see below); i.e., inserting the screen makes the beam behave like it has a shot noise distribution. The screen is approximately $20 \mathrm{~cm}$ upstream of the undulator, which is too close to fully reset the beam to a random distribution, but comparing screenIN and screen-OUT radiation gives a second means to estimate the true shot noise level. Figure 4 shows screen-IN data taken at several values of $R_{56}$. In each case, inserting the screen moves the output closer to the expected shot noise level, but some correlated effects from both subradiance and superradiance remain with the screen inserted. Nevertheless, the screen-IN data with $R_{56}=0.1 \mathrm{~mm}$ gives a good approximation of the shot noise level. We also note that this is a conservative estimate; if the true shot noise level is higher, then the quoted degree of suppression is actually an underestimate.

\section{LIMITS TO OBSERVED SUPPRESSION}

With the gun phase at -14 degrees and $R_{56}=2.2 \mathrm{~mm}$, we observe a factor of 2.6 reduction in the integrated farfield radiation. In the simple 1D model, the noise factor nearly reaches 0 for $\Upsilon=1$, with the maximum level of suppression limited by fundamental beam properties of emittance and energy spread [10,32,35]. In practice, a number of other longitudinal and transverse effects also inhibit full suppression. First, because different parts of the beam have different values of $n_{0}$, there is no single value of $R_{56}$ that suppresses the entire beam. (See e.g., [12,35] for a discussion of a Gaussian distribution in a short interaction.) The beam is also expected to have strong peak current dependence along the longitudinal axis. Our diagnostic is 
not time resolved, so the measured radiation is integrated across the entire bunch length and at any given $R_{56}$ includes portions of the beam at different positions along the quadratic suppression curve. Indeed, nonuniform current may explain the observed radiation reduction factors of only 2.1 ( -19.5 degrees) and 1.6 ( -5 degrees) for the other gun phase settings. If we were able to either produce a more uniform phase space (e.g., with a linearizing cavity) or make a time-resolved measurement, we would expect even stronger suppression. We note that for applications such as seeding FELs, suppression is only relevant in the core of the beam, both longitudinally and transversely, where it should be possible to achieve higher levels of suppression.

\section{ANGULAR DEPENDENCE}

The degree of suppression also depends on the angle of observation, $\theta$, relative to the motion of the electron beam. Indeed, previous OTR measurements observed weak suppression partially because of transverse form factors that are not included in Eq. (1) [12]; at large angles, $\theta>\theta_{\max }=$ $\lambda_{r} /\left(2 \pi \sigma_{u}\right)$ (for beam size in the undulator $\sigma_{u}$ ), the radiation is no longer transversely coherent and suppression disappears (see e.g., [33]). The experimental setup of [12] could not isolate the on-axis radiation, which limited the observed level of suppression in that study.

In this experiment we measure the far-field radiation to determine the angular radiation profile. Undulator radiation has a wavelength dependence at angle $\theta$ of

$$
\lambda_{r}(\theta)=\frac{\lambda_{u}}{2 \gamma^{2}}\left(1+K^{2} / 2+\gamma^{2} \theta^{2}\right) .
$$

With $\lambda_{r}(0)=800 \mathrm{~nm}$, and camera efficiency $50 \%$ lower at $890 \mathrm{~nm}$, we only observe the radiation out to approximately $\theta=2 \mathrm{mrad}$. With undulator parameter $K=1.8$, radiation extends vertically beyond $\theta=2 \mathrm{mrad}$, so the camera cutoff affects both planes equally and we observe a round beam in Fig. 2. Assuming beam size in the undulator of $\sigma_{u}=100 \mu \mathrm{m}$, suppression should only be observed out to $\theta_{\max } \approx 1.5 \mathrm{mrad}$. As a result, we would expect there to be an angular dependence in the radiation profile from the noise-suppressed beam as compared with the shot-noise dominated beam. Figure 5(a) shows the angular dependence of the -14 deg case, normalized by the shot-noise dominated $R_{56}=0.1 \mathrm{~mm}$ measurement. There is slightly more suppression at smaller angles, but it is a weaker effect than expected, suggesting either $\sigma_{u}$ is smaller than anticipated in the undulator, or that the transverse coherence length is smaller than the beam size. As a check of the theory, we repeat the analysis with the OTR screen inserted, which increases the beam size on the order of $100 \mu \mathrm{m}$ due to scattering. Though longitudinal smearing also reduces the on-axis suppression, Fig. 5(b) shows clear angular dependence in the remaining suppression with the screen inserted, consistent with a beam size above $100 \mu \mathrm{m}$. It is

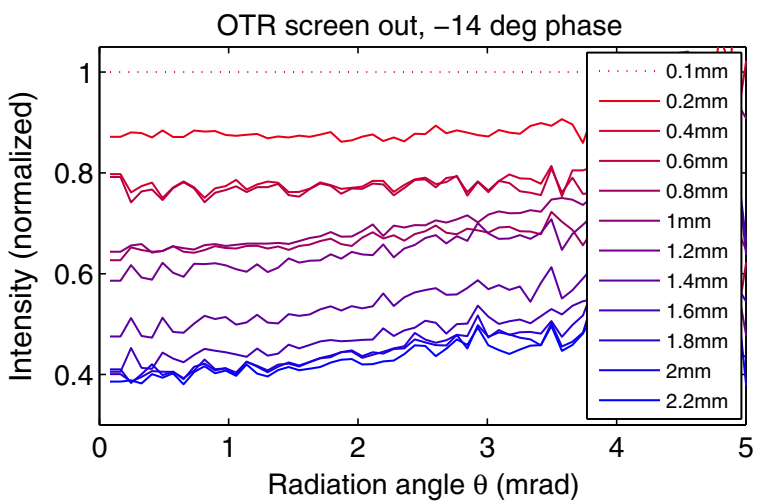

(a)

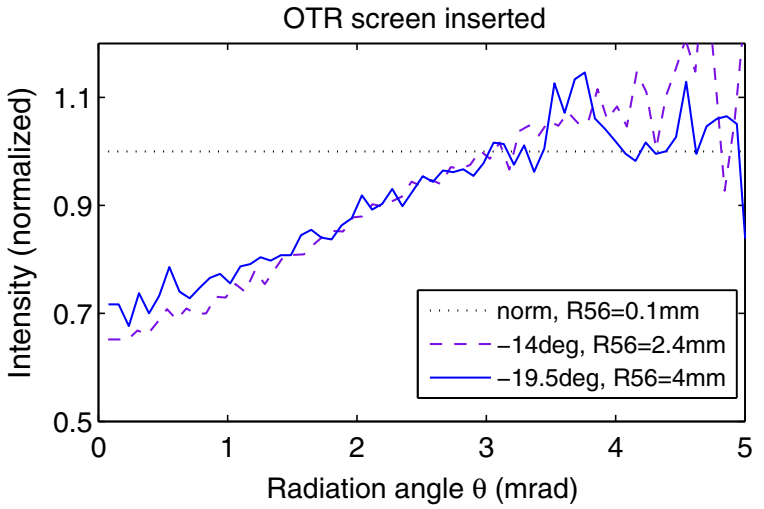

(b)

FIG. 5. Radiation intensity as a function of angle from direction of motion normalized by the measurement with $R_{56}=0.1 \mathrm{~mm}$. (a) Case of $-14 \mathrm{deg}$ gun phase for different values of $R_{56}$. Suppression is slightly stronger on axis, but is relatively uniform across the FWHM beam (2 mrad). (b) Inserting an OTR screen increases emittance, which amplifies angular effects. At optimal suppression for both -14 deg and -19 deg gun phases, the degree of suppression is angle dependent, with suppression predominantly on axis. Data for the -14 deg case corresponds to the red square, $R_{56}=2.4 \mathrm{~mm}$ in Fig. 4 .

worth noting spontaneous radiation at large angles is not relevant to the FEL process, so a lack of wide angle suppression is likely not a concern for seeding applications.

To combine undulator subradiance with external FEL seeding, both the interaction and dispersive regions should be as close as possible to the undulator; emittance effects and energy spread, as well as plasma oscillations, can reduce the degree of suppression over long distances. In most seeding configurations a chicane immediately upstream of the undulator makes room for a mirror to reflect the external laser into the undulator line (see e.g., Fig. 1 in [18]). This mirror chicane can be tuned to minimize spontaneous radiation, or if the optimal $R_{56}$ of suppression is too small to fit the mirror, then a weak, compact chicane can be placed between the mirror chicane and the undulator. The optimal $R_{56}$ depends on machine 
parameters [10], but at the higher peak currents associated with FELs, subradiance would occur at lower $R_{56}$ values than used here.

\section{CONCLUSION}

This paper presents the first demonstration of subradiant spontaneous undulator radiation. Using the dispersive method to suppress electron beam shot noise, we observe a suppression factor of up to 2.6 in the time and angle integrated far-field radiation from an undulator. The core of the beam, which supports the bulk of the high-gain FEL amplification process, is expected to have even stronger suppression. The substantial level of suppression demonstrated here should allow, among other applications, seeding of an FEL with lower pulse energy, thus extending seeding towards the $\mathrm{x}$-ray regime. Future work will extend these results to shorter wavelengths as well as look to combine subradiance with external seeding of an FEL.

\section{ACKNOWLEDGMENTS}

This work is supported by the U.S. DOE Office of Basic Energy Sciences under Contract No. DE-AC0276SF00515. The experimental work was conducted at the Next Linear Collider Test Accelerator (NLCTA) facility, which is partly supported by U.S. DOE Office of High Energy Physics. We acknowledge partial support by the U.S.-Israel Binational Science Foundation (BSF) Jerusalem, Israel.

[1] R. H. Dicke, Phys. Rev. 93, 99 (1954).

[2] W. Zhou and T. W. Odom, Nat. Nanotechnol. 6, 423 (2011).

[3] T. Bienaime, N. Piovella, and R. Kaiser, Phys. Rev. Lett. 108, 123602 (2012).

[4] Y. Takasu, Y. Saito, Y. Takahashi, M. Borkowski, R. Ciurylo, and P. S. Julienne, Phys. Rev. Lett. 108, 173002 (2012).

[5] B. H. McGuyer, M. McDonald, G. Z. Iwata, M. G. Tarallo, W. Skomorowski, R. Moszynski, and T. Zelevinsky, Nat. Phys. 11, 32 (2014).

[6] R. Bonifacio, B. W. J. McNeil, and P. Pierini, Phys. Rev. A 40, 4467 (1989).

[7] A. Gover, Phys. Rev. ST Accel. Beams 8, 030701 (2005).

[8] A. Gover and E. Dyunin, Phys. Rev. Lett. 102, 154801 (2009).

[9] V. Litvinenko, in Proceedings of the 31st International Free Electron Laser Conference (FEL 09), Liverpool, UK (STFC Daresbury Laboratory, Warrington, 2009).
[10] D. Ratner, Z. Huang, and G. Stupakov, Phys. Rev. ST Accel. Beams 14, 060710 (2011).

[11] G. Stupakov, A. Sessler, and M. S. Zolotorev, in Proceedings of COOL'13 (Murren, Switzerland, 2013), p. THAM2HA03.

[12] D. Ratner and G. Stupakov, Phys. Rev. Lett. 109, 034801 (2012).

[13] A. Gover, A. Nause, E. Dyunin, and M. Fedurin, Nat. Phys. 8, 877 (2012).

[14] E. Allaria et al., Nat. Photonics 7, 913 (2013).

[15] L. H. Yu, Phys. Rev. A 44, 5178 (1991).

[16] I. Ben-Zvi, L. F. D. Mauro, S. Krinsky, M. G. White, and L. H. Yu, Nucl. Instrum. Methods Phys. Res., Sect. A 304, 181 (1991).

[17] G. Stupakov, Phys. Rev. Lett. 102, 074801 (2009).

[18] D. Xiang, E. Colby, M. Dunning, S. Gilevich, C. Hast, K. Jobe, D. McCormick, J. Nelson, T. O. Raubenheimer, K. Soong, G. Stupakov, Z. Szalata, D. Walz, S. Weathersby, M. Woodley, and P.-L. Pernet, Phys. Rev. Lett. 105, 114801 (2010).

[19] G. Lambert et al., Nat. Phys. 4, 296 (2008).

[20] R. Bonifacio, C. Pellegrini, and L. M. Narducci, Opt. Commun. 50, 373 (1984).

[21] A. Gover and E. Dyunin, IEEE J. Quantum Electron. 46, 1511 (2010).

[22] V. N. Litvinenko and Y. S. Derbenev, Phys. Rev. Lett. 102, 114801 (2009).

[23] D. Ratner, Phys. Rev. Lett. 111, 084802 (2013).

[24] M. Borland et al., Nucl. Instrum. Methods Phys. Res., Sect. A 483, 268 (2002).

[25] E. L. Saldin, E. A. Schneidmiller, and M. V. Yurkov, Nucl. Instrum. Methods Phys. Res., Sect. A 490, 1 (2002).

[26] Z. Huang, M. Borland, P. Emma, J. Wu, C. Limborg, G. Stupakov, and J. Welch, Phys. Rev. ST Accel. Beams 7, 074401 (2004).

[27] R. Akre et al., Phys. Rev. ST Accel. Beams 11, 030703 (2008).

[28] V. A. Goryashko and V. Ziemann, Phys. Rev. ST Accel. Beams 16, 030702 (2013).

[29] Z. Huang, J. Wu, and T. Shaftan, ICFA Beam Dynamics Newsletter 38, 37 (2005).

[30] D. Xiang et al., Phys. Rev. Lett. 108, 024802 (2012).

[31] A. Gover, E. Dyunin, T. Duchovni, and A. Nause, Phys. Plasmas 18, 123102 (2011).

[32] A. Nause, E. Dyunin, and A. Gover, Phys. Plasmas 21, 083114 (2014); 21, 083114(E) (2014).

[33] A. Chao and M. Tigner, Handbook of Accelerator Physics and Engineering (World Scientific, Singapore, 2006).

[34] P. Emma, K. Bane, M. Cornacchia, Z. Huang, H. Schlarb, G. Stupakov, and D. Walz, Phys. Rev. Lett. 92, 074801 (2004).

[35] D. Ratner, Much ado about microbunching: Coherent bunching in high brightness electron beams, Ph.D. thesis, Department of Applied Physics, Stanford University, 2011. 